\title{
Técnicas diferentes para eliminar la capa de resina inhibida por oxígeno, en un composite nanohíbrido sometido a desgaste abrasivo
}

\section{Different techniques to eliminate the layer of resin inhibited by oxygen, in a nanohybrid composite subjected to abrasive wear}

\section{Diferentes técnicas para eliminar a camada de resina inibida pelo oxigênio, em um composto nanohíbrido submetido a desgaste abrasivo}

\author{
Javier E. Sánchez-Sánchez \\ javieritosanchez@gmail.com Katherine \\ G. Rodríguez-Cervantes \\ kathegrc@gmail.com \\ Ana del Carmen Armas \\ ana_del_ec@yahoo.es Iván \\ R. García-Merino \\ ivangarch@usp.br \\ Henry S. Oñate-Negrete \\ hson1987@hotmail.com
}

Recibido: 15 de septiembre de 2017 * Corregido: 12 de noviembre de 2017 * Aceptado: 8 de enero de 2017

I Odontólogo, Estudiante Especialidad de Cirugía y Traumatología Bucomaxilofacial, Universidad de Sao Paulo, Rio Pequeño, Sao Pauolo, Brazil.

II Odontóloga, Estudiante Especialidad de Ortodoncia. Universidad Central del Ecuador, Quito, Ecuador.

III Phd en Operatoria Dental, Docente de la Universidad Central del Ecuador, Quito, Ecuador.

IV Diploma Superior en Implantes Odontológicos, Especialista en Prótesis Bucal Fija, Doctor en Odontología, Docente de la Universidad Central del Ecuador, Quito, Ecuador.

v Odontólogo, Universidad Central del Ecuador, Quito, Ecuador. 


\section{Resumen}

Este trabajo buscó evaluar la eficiencia de tres técnicas diferentes utilizadas para eliminar la capa de resina inhibida, los especímenes fueron sometidos a un pesaje inicial y luego a un desgaste abrasivo por cepillado in vitro por un lapso de 6 horas, después de lo cual se determinó el peso final y los datos obtenidos fueron comparados y analizados estadísticamente. Fueron confeccionados 48 muestras de resina nanohíbrida Brilliant (Coltene) color A2 esmalte; divididas en 4 grupos de 12 especímenes cada uno. Al primer grupo no se lo sometió a ningún protocolo para eliminar la capa de resina inhibida (grupo control); el segundo grupo se aplicó una ligera presión con una matriz de celulosa en la superficie de la resina y luego se la fotocuró; el tercer grupo se procedió con un protocolo de pulido después de la fotopolimerización de la resina, y el cuarto grupo se utilizó un compuesto a base de glicerina en la superficie de la resina y se la fotocuró. Como resultado, estadísticamente las pruebas t Student y Kruskal Wallis permitieron determinar $\mathrm{p}=0.002$, que evidencia diferencia entre los grupos. Se concluye que el empleo de matriz de celulosa durante el proceso de eliminación de capa inhibida permitió obtener la mejor estabilidad en cuanto a las mediciones de peso.

Palabras clave: capa de resina inhibida; desgaste; resina compuesta.

\section{Abstract}

This work sought to evaluate the efficiency of three different techniques used to remove the inhibited resin layer, the specimens were subjected to initial weighing and then to abrasive wear by brushing in vitro for a period of 6 hours, after which The final weight was determined and the data obtained were compared and statistically analyzed. 48 samples of nanohybrid resin Brilliant (Coltene) color A2 enamel; divided into 4 groups of 12 specimens each. The first group was not subjected to any protocol to remove the inhibited resin layer (control group); The second group applied a light pressure with a cellulose matrix on the surface of the resin and then it was photocurlowed; The third group proceeded with a polishing protocol after photopolymerization of the resin, and the fourth group used a glycerinbased compound on the surface of the resin and photocuring it. As results Statistically, Student $t$ and Kruskal Wallis tests allowed us to determine $\mathrm{p}=0.002$, which showed a difference between the

\footnotetext{
20 Vol. 4, núm. 2, abril, 2018, pp. 20-33 Javier E. Sánchez Sánchez, Katherine G. Rodríguez Cervantes, Ana del Carmen Armas, Iván R. García Merino, Henry S. Oñate-Negrete
} 
groups. To conclude that the use of cellulose matrix during the process of removal of inhibited layer allowed to obtain the best stability in terms of weight measurements.

Keywords: Inhibited resin layer; wear; composite resin.

\section{Resumo}

Este estudo destinado a avaliar a eficácia de três diferentes técnicas utilizadas para remover a camada de resina de espécimes inibidos foram submetidas a uma primeira pesagem e, em seguida, a um desgaste abrasivo por escovagem in vitro durante um período de 6 horas, após o que foi determinada $\mathrm{O}$ peso final e os dados obtidos foram comparados e analisados estatisticamente. Foram confeccionadas 48 amostras de esmalte A2 de resina nanohíbrida brilhante (Coltene); dividido em 4 grupos de 12 espécimes cada. O primeiro grupo não foi submetido a nenhum protocolo para eliminar a camada de resina inibida (grupo controle); no segundo grupo foi aplicada uma ligeira pressão com uma matriz de celulose na superfície da resina e depois fotocurada; o terceiro grupo foi submetido a um protocolo de polimento após a fotopolimerização da resina, e o quarto grupo utilizou um composto à base de glicerina na superfície da resina e fotopolimerizando-a. Como resultado, estatisticamente os testes Student $t$ e Kruskal Wallis permitiram a determinação de $p=0,002$, o que evidencia a diferença entre os grupos. Conclui-se que o uso da matriz de celulose durante o processo de eliminação da camada inibida permitiu obter a melhor estabilidade em termos de medidas de peso.

Palavras chave: Camada de resina inibida; desgaste; resina composta.

\section{Introducción}

El tratamiento restaurador de las lesiones cariosas consiste en la eliminación del tejido dental que fue dañado de forma irreversible, buscando que el tejido remanente permanezca sano, recuperando la morfología, función y estética perdidas (Heminwag y col, 2006), buscando la máxima preservación y mínima intervención (Jung, 2005), contando con numerosos biomateriales directos e indirectos de uso odontológico, de diferentes características y propiedades (Litonja, 2005), escogidos de acuerdo a la situación clínica presente en el paciente (Machado y col, 2011).

\footnotetext{
21 Vol. 4, núm. 2, abril, 2018, pp. 20-33 Javier E. Sánchez Sánchez, Katherine G. Rodríguez Cervantes, Ana del Carmen Armas, Iván R. García Merino, Henry S. Oñate-Negrete
} 
En la actualidad, existe un anhelo compartido por parte del profesional y pacientes por el uso de materiales restauradores estéticos, prácticos y seguros (Pontons y col, 2010), siendo el composite el biomaterial más empleado en restauraciones directas (Ratto y col, 2008), resultado de una mezcla de cargas inorgánicas silanizadas con un monómero acrílico polimerizado para formar una restauración sólida, conformada por tres fases, orgánica o matriz, dispersa o de relleno e interfacial o de unión establecida por agentes silano (Rodríguez y col, 2010), con propiedades mecánicas atribuidas a la parte orgánica del material responsable de la contracción volumétrica y la tensión (Soparkar y col, 2004), que requieren de un tratamiento previo de la superficie dental para conseguir su adhesión (Teixeira, 2005), presentando en este proceso contracción de polimerizado y cambio dimensional térmico (Versteeg y col, 2008).

La fase dispersa o de relleno del composite representada por partículas obtenidas por molienda o de compuestos de silicio (Infeld, 2008), sílice, bario, estroncio (Cury y col, 2004) responsables de la dureza y de la resistencia a la fractura del material (Rodríguez y col, 2010) se presentan entre un 52\% a $88 \%$ en el material (Sabbagh y col, 2004), a estos elementos se suman agentes acopladores de las fases, titanatos, circonatos, silanos orgánicos (Kuijs y col, 2003), formadores de enlaces covalentes con la resina cuando ésta polimeriza (De Sousa y col, 2005), ayudando a mejorar la estabilidad hidrolítica para prevenir la penetración de agua a través de la interfase relleno-matriz (Braga, 2004).

El fotoiniciador más comúnmente empleado en la

$\mathrm{s}$ resinas compuestas es la canforoquinona (Skrtic, 2000), presente en 0.2\% de peso (Terry, 2004), con una sensibilidad de $468 \mathrm{~nm}$ dentro del espectro de la longitud de onda azul (Wong, 2004), a los que se suma el hidroxitolueno butilado, la benzoquinona, la hidroquinona y derivados del fenol como elementos inhibidores (Soderholm y col, 2001). Varios factores son los relacionados con el desgaste de la resina, uno de ellos al observarse el aumento en el porcentaje de relleno dentro de la resina compuesta disminuye el desgaste ( Heintze y col, 2005), notándose que cuanto menos sea el tamaño

\footnotetext{
22 Vol. 4, núm. 2, abril, 2018, pp. 20-33 Javier E. Sánchez Sánchez, Katherine G. Rodríguez Cervantes, Ana del Carmen Armas, Iván R. García Merino, Henry S. Oñate-Negrete
} 
de las partículas menor será la susceptibilidad al desgaste, de ahí que el menor desgaste se percibe en las resinas de microrrelleno y los híbridos de partícula submicrónica (Heminwag y col, 2006), desgaste al que se le atribuye a la producción de microfracturas en la masa de la resina ( Cury y col, 2004), producida por cargas repetidas, que inician en grietas que crecen y confluyen desencadenando el desprendimiento de partículas de relleno de la superficie del material, la sorción química y la degradación hidrolítica de los agentes de enlace (Imfeld, 2008).

En el medio bucal, el agua y otros componentes que se encuentran en la saliva actúan degradando y desprendiendo el relleno de la superficie de los composites fenómeno denominado como "plucking out" (Hux y coll, 2003), desencadenando la aparición de irregularidades en la superficie de la restauración, en dependencia del tamaño de la partícula( Mitra y col, 2003), observándose que composites con cargas más grandes presentan mayor pérdida de peso y la mayor aspereza tras procedimientos de cepillado.

La abrasión considerada como el desgaste mecánico asociado a elementos no dentarios, desencadena real importancia al considerar el cepillado dental como práctica habitual en el paciente (Soderholm y col, 2001), observándose consecuencias en dependencia de la fuerza con que se ejecuta, tiempo, fuerza aplicada, sitio de la arcada dentaria donde empieza el cepillado y factores inherentes al material utilizado para la higiene dental, el tipo de material, dureza, características de las cerdas, flexibilidad, largo del mango del cepillo, el poder abrasivo, el pH y la cantidad de dentífrico utilizado.

Evaluaciones realizadas sobre resinas compuestas, considerando su color y tiempo de polimerización, revelaron similar capacidad de desgaste entre los materiales, relacionando la susceptibilidad al desgaste de una resina con el grado de dureza en la superficie de esta, condicionado por la absorción de agua, la rugosidad y en primer lugar en grado de importancia por el grado de conversión de sus monómeros (Kuijs y col, 2003). Sugiriendo que, en condiciones sanas, las sustancias abrasivas no deberían tener ningún efecto nocivo (Heminwag y col, 2006), en la actualidad el abrasivo de uso común es el sílice (SiO2) (Versteeg y col, 2008), los humectantes como el glicerol, sorbitol y xilitol (dulces) y propilenglicol presentes entre el $20-40 \%$, son sustancias que impiden la pérdida de agua

\footnotetext{
23 Vol. 4, núm. 2, abril, 2018, pp. 20-33 Javier E. Sánchez Sánchez, Katherine G. Rodríguez Cervantes, Ana del Carmen Armas, Iván R. García Merino, Henry S. Oñate-Negrete
} 
(Braga, 2004), excipientes como el agua destilada o desionizada, conforman del 20\% al 35\% del dentífrico.

A esta composición se suman, los estabilizadores, impiden que la fase sólida y la fase líquida se separen y son coloides hidrofílicos como la goma arábiga, la goma de Kara, coloides de algas marinas, metilcelulosa y carboximetilcelulosa, los detergentes como laurilsulfato de sodio, N-lauril sarcosinato de sodio, sodio alkil sulfoacetato y sulfoacetato alcalino de sodio sonencargados de disminuir la tensión superficial (Hux y col, 2003), saborizantes empleados para mejorar la aceptación de un dentífrico por el público y finalmente los preservantes del tipo benzoatos, diclorofenol, formaldehidos e hidroxibenzoatos que contribuyen en la protección de los humectantes y aglutinantes del ataque de las bacterias y del crecimiento de los hongos (Cury y col, 2004).

Considerando el empleo generalizado de composites y la composición de un dentífrico, este estudio pretende evaluar los cambios que se producen en relación a la pérdida de peso en la superficie de resina, por la acción de un proceso abrasivo en relación al grado de dureza de una resina compuesta nanohíbrida.

\section{Materiales y métodos}

Se plantea un estudio experimental, donde 48 fragmentos de resina, de $0.7 \mathrm{~cm}$. de diámetro y de 0.2 $\mathrm{cm}$. de profundidad, de marca Brilliant (Coltene) color A2 Esmalte, con especificaciones técnicas referentes a diámetro medio de las partículas de relleno $0.6 \mu \mathrm{m}$; la distribución de las partículas de relleno oscila entre $0.01-2.5 \mu \mathrm{m}$; el contenido de relleno por volumen es de $65 \%$ y el contenido de relleno en peso es de $80 \%$, construidos en base a una matriz de dimensiones pre establecidas colocando la resina de manera incremental de 2,0 mm polimeriznado por 20 segundos (Kuijs y col, 2003).

Fueron establecidos cuatro grupos, uno de control y tres experimentales, al experimental no se les aplicó ningún método para eliminar la capa de resina inhibida; un grupo al que se le aplicó una ligera

\footnotetext{
24 Vol. 4, núm. 2, abril, 2018, pp. 20-33 Javier E. Sánchez Sánchez, Katherine G. Rodríguez Cervantes, Ana del Carmen Armas, Iván R. García Merino, Henry S. Oñate-Negrete
} 
presión de celulosa en la superficie de resina, como método para eliminar la capa de resina inhibida fotopolimeriznado la resina a seguir, un grupo al que tras la fotopolimerización sin colocar ningún aditamento previo fueron sometidos a pulido como método para eliminar la capa de resina inhibida; un grupo al que tras colocar resina se le aplicó glicerina $\mathrm{DeOx}{ }^{\circledR}$ de la casa Ultradent en la superficie de su última capa y después fotopolimerizado, padronizando una distancia de $2 \mathrm{~cm}$ entre la superficie de resina y la punta del aparato fotopolimerizador Led B Woodpecker; realizándose el pulido posterior mediante Astropol® (Ivoclar Vivadent) de forma secuencial tras verificar que los fragmentos se encuentren íntegros y sin la presencia de burbujas, cada fragmento fue evaluado en cuanto a su peso en balanza de precisión (GM series Professional Digital Jewelry Scale).

Todos los fragmentos fueron sometidos a un proceso de desgaste por abrasión en una máquina cepilladora, por un lapso de 6 horas correspondientes a 91800 ciclos, (un ciclo es el movimiento completo de vaivén del cepillo), tomando en cuenta que el tiempo promedio del cepillado oral de un individuo es de casi 2 minutos (Pontons y col, 2010), es decir que se ejecutan aproximadamente alrededor de 340 ciclos en los 2 minutos, definimos a un ciclo como el movimiento completo de vaivén del cepillo; esto se realiza con una velocidad regular (no muy rápida, ni muy lenta). Para conservar un estado de salud bucal una persona debe cepillar sus dientes 3 veces al día (según instructivo de Colgate), lo que equivale a 6 minutos diarios, 180 minutos al mes y 540 minutos a los tres meses. Considerando que un cepillo dental debe estar alrededor de los tres meses en uso, dando un total de 91800 ciclos ejecutados en 6 horas en una máquina de motor marca Weg de $1 / 4$ HP $(0.18$ $\mathrm{KW}$ ), de $110 \mathrm{~W}$, frecuencia de $60 \mathrm{HZ}$, una intensidad de $5.00 \mathrm{~A}$, que produce $1730 \mathrm{rpm}$; velocidad que fue modificada con dos piñones, uno grande y otro pequeño, para generar la velocidad aproximada de $270 \mathrm{rpm}$ y producir el movimiento de vaivén de los cepillos Colgate $360^{\circ}$ con una fuerza equivalente a 0.333 libras, en combinación con pasta dental Colgate Ultra Blanco sobre los diferentes fragmentos sometiéndoles al concluir este proceso a la ejecución del pesaje respectivo de cada uno de los fragmentos (De Sousa y col, 2003).

\footnotetext{
25 Vol. 4, núm. 2, abril, 2018, pp. 20-33 Javier E. Sánchez Sánchez, Katherine G. Rodríguez Cervantes, Ana del Carmen Armas, Iván R. García Merino, Henry S. Oñate-Negrete
} 
La cantidad de pasta dental que fue agregada al respectivo grupo fue en una cantidad de 2 gramos, que representa la cantidad de pasta dental que cubre la longitud de la cabeza del cepillo dental; ésta cantidad se colocó cada hora durante las 6 horas que duró el proceso abrasivo, ya que, en el transcurso de una hora, con el movimiento de vaivén, la pasta dental se dispersó, quedando sin ella la superficie de resina. Al concluir el proceso abrasivo con la máquina cepilladora, se llevó a todos los fragmentos de resina a la balanza de precisión y se tomó el peso final de cada uno, los datos fueron recolectados en una tabla específicamente diseñada para el efecto (Braga, 2004).

\section{Análisis de los resultados}

El análisis de la información obtenida se realizó a través de la prueba t Student, la cual se utiliza para la determinación de las diferencias entre dos medidas muestrales, y la prueba de Kruskal Wallis la cual se la emplea cuando se quiere remplazar tres o más poblaciones, pero sin requerir un supuesto de normalidad. Las matrices para los tres primeros grupos (pulido, matriz de celulosa, pulido) se presentan como homogéneas (peso inicial similar), salvo en el grupo donde se aplicó glicerina que presentó un valor inicial más alto en cuanto a su peso. Otra tendencia visible es que con el grupo sometido a proceso de pulido y con el grupo al cual se aplicó glicerina, se notaron pérdidas de peso luego del cepillado. Los grupos: control y matriz de celulosa respectivamente presentaron un leve aumento de peso.

Los datos fueron sometidos a un análisis estadístico a través de $\mathrm{t}$ Student demostraron variaciones que resultaron significativas $(\mathrm{p}<0,05)$ para los grupos tratados con pulido previo y con glicerina. Se observaron diferencias significativas para los grupos control y matriz de celulosa, realizando evaluaciones entre el peso inicial y final, como se observa en tabla 1.

\begin{tabular}{|l|l|r|c|c|}
\hline \multirow{2}{*}{ GRUPO } & PESO & Media & $\begin{array}{c}\text { Diferencia } \\
\text { medias }\end{array}$ & p \\
\hline \multirow{2}{*}{ Control } & INICIAL & 120,17 & & \\
\cline { 2 - 3 } & FINAL & 122,33 & 2,2 & \multirow{2}{*}{, 287} \\
\hline
\end{tabular}

26 Vol. 4, núm. 2, abril, 2018, pp. 20-33 Javier E. Sánchez Sánchez, Katherine G. Rodríguez Cervantes, Ana del Carmen Armas, Iván R. García Merino, Henry S. Oñate-Negrete 
Técnicas diferentes para eliminar la capa de resina inhibida por oxígeno, en un composite nanohíbrido sometido a desgaste abrasivo

\begin{tabular}{|l|l|r|r|r|}
$\mathbf{M}$ & INICIAL & 121,58 & & \\
\cline { 2 - 3 } Celulosa & FINAL & 122,83 & 1,3 &, 128 \\
\cline { 2 - 3 } & INICIAL & 120,75 & & \\
\cline { 2 - 3 } Glicerina & FINAL & 114,83 & $-5,9$ &, 012 \\
\cline { 2 - 3 } & INICIAL & 127,67 & & \\
\cline { 2 - 3 } & FINAL & 124,50 & $-3,2$ &, 009 \\
\hline
\end{tabular}

Tabla 1. Variaciones entre peso inicial y final de cada grupo

Fuente: elaboración propia.

El grupo en el que se registró mayor pérdida fue en el que se pulió previamente, y otro grupo en el que se registró pérdida fue en el que se aplicó glicerina. Dado que en algunos casos se registró aumento de peso y en otras pérdidas de peso, se consideró conveniente realizar la prueba de Kruskal Wallis, mediante el programa estadístico SPSS, se determinó un p de 0,002, con lo que se pudo concluir que existen diferencias significativas en la variación del peso para los cuatro grupos.

\section{Discusión de los resultados}

La evolución a lo largo de las últimas décadas en cuanto a conocimientos en materia de Cariología y Periodoncia, han hecho que para el control mecánico del biofilm, se desarrollen numerosas técnicas de cepillado dental sin tener evidencia de que técnica es superior a otra, coincidiendo con otros autores (Terry, Geller, 2004) considerando que el método ideal de cepillado es el que permite acceder y limpiar eficientemente la mayoría de áreas de la boca en menor tiempo y sin causar ninguna lesión a los tejidos (Versteeg y col, 2008), siendo el método de cepillado horizontal el más utilizado por quienes nunca recibieron instrucciones acerca de la higiene bucal (Rodríguez y col, 2008).

Se puede añadir que no existe un método de cepillado universal que favorezca a todos los pacientes, que cumpla con los medios que satisfaga al odontólogo, y que a la vez cumpla con las necesidades de salud del paciente. Sin embargo, el método ideal de cepillado más acorde a la rutina de limpieza bucal estará en relación: con el desarrollo de la motricidad fina y con una coordinación efectiva entre la

\footnotetext{
27 Vol. 4, núm. 2, abril, 2018, pp. 20-33 Javier E. Sánchez Sánchez, Katherine G. Rodríguez Cervantes, Ana del Carmen Armas, Iván R. García Merino, Henry S. Oñate-Negrete
} 
mano y el ojo del operador (Versteeg y col, 2008). En cuanto a la fuerza utilizada durante el cepillado, es necesario mencionar que la aplicación de una fuerza exagerada provocaría traumatismos en los tejidos gingivales (Soderholm y col, 2001) de allí que se recomienda emplear una fuerza no mayor a 150 gf., que equivaldría a un cepillado con las cerdas sin flexionar, logrando con ello una limpieza adecuada.

Actualmente, los conceptos de salud bucal giran en torno al control de la placa bacteriana mediante el uso de pastas y cepillos dentales, los cuales se presentan en el mercado en diferentes colores y texturas y en el caso particular de los dentífricos, en diferentes sabores. Sin embargo, se ha dejado de lado el efecto abrasivo de esta combinación como responsable del deterioro de los composites, tal vez por suponer que su efecto sobre la superficie es mínimo o por responsabilizar a un solo factor como el causante de este tipo de desgaste. En este estudio se empleó la técnica de cepillado horizontal (De Sousa y col, 2005), es la más manejada debido a su fácil ejecución. La búsqueda por una sonrisa perfecta ha hecho que los dentistas empleen procedimientos restauradores estéticos, para lo cual se emplean materiales restauradores del tipo resina compuesta, los cuales son usados especialmente en el sector anterior permitiendo obtener buenos resultaos en cuanto a estética y funcionalidad pero con una duración limitada, esto se ha atribuido a hábitos dañinos o problemas oclusales por parte del paciente, fallas del propio material o la aplicación de una técnica deficiente por parte del operador (Hemingway y col, 2006).

En cuanto a protocolos para la utilización de las resinas compuestas, no existe un consenso sobre cuál sería la mejor técnica para eliminar la capa de resina inhibida, es mas no se cuenta con artículos científicos que orienten al lector hacia la utilización de un método o técnica en particular. Con estos antecedentes, este trabajo pretendió evaluar la acción de tres diferentes técnicas para eliminar la casa de resina inhibida en una resina compuesta nanohíbrida, los segmentos de esta resina fueron sometidos a un proceso abrasivo con el fin de comprobar la eficiencia de cada una de las técnicas; analizando el cambio en la masa de cada uno de los fragmentos antes y después del proceso abrasivo. Se optó por

\footnotetext{
28 Vol. 4, núm. 2, abril, 2018, pp. 20-33 Javier E. Sánchez Sánchez, Katherine G. Rodríguez Cervantes, Ana del Carmen Armas, Iván R. García Merino, Henry S. Oñate-Negrete
} 
la evaluación en el cambio de peso de cada uno de los fragmentos, para interpretar a partir de la variación entre el peso inicial y final, la eficacia de la técnica aplicada.

En el grupo control el aumento de peso fue muy significativo, y en tres de las muestras existió pérdida de peso, por lo que se plantea que en este grupo a más de sorción de agua hubo una solubilidad de los componentes del composite expresando así un fracaso de la técnica. En cuanto a la sorción de agua, esta pudo haber llevado al composite a la pérdida de partículas de relleno por degeneración del agente silano, el cual juega un papel importante en la detención de la degeneración hidrolítica de la resina (Litonjua y col, 2005). La deposición del agente silano entre la matriz y el relleno se realiza en multicapas, las capas más externas del silano son muy sensibles y se deterioran más rápidamente en comparación con las internas que son química e hidrolíticamente más estables que las internas. Cuando el agua se aproxima al relleno se neutraliza la energía superficial del mismo, facilitando la penetración del agua en su interior (Braga, 2004). La presencia de poros en la superficie de la resina que pudo haber por defectos de manipulación del material o por no utilizar ningún método de pulido favoreció a la sorción de agua (Teixeira, 2005), desencadenando solubilidad que se produce cuando las resinas se ponen en contacto con un medio húmedo y por efecto de la energía cinética de las moléculas del líquido se arrancan partículas de la superficie (Rodríguez y col, 2008).

En los resultados del pesaje y la variación del peso de los especímenes del grupo sometido a matriz de celulosa, se puede notar que casi el $67 \%$ de los especímenes aumentaron de peso, dicho aumento se expresó de forma no muy significativa, lo que indica que la resina aumento de peso, presuntamente por sorción de agua, por encontrarse en un medio húmedo el cual era promovido por la pasta dental y el agua destilada usados para el proceso abrasivo.

Dicha sorción de agua está promovida por la fase matriz de la resina; esto produce un debilitamiento en las propiedades mecánicas del composite asociado a la degeneración hidrolítica de las uniones matriz-partícula, además de generar degradaciones físico-químicas en la matriz y en el relleno desencadenando aumento de peso de los especímenes pertenecientes a este grupo aparentemente no puede ser interpretado como un éxito de la técnica practicada, sino más bien queda por comprobar si

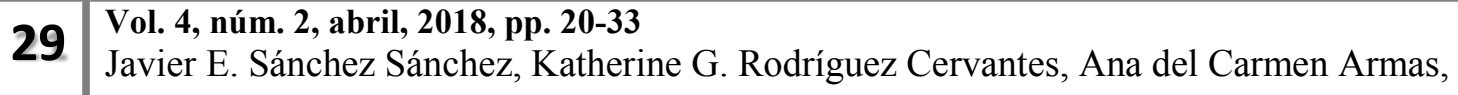
Iván R. García Merino, Henry S. Oñate-Negrete
} 
la técnica se la acogería como un éxito o un fracaso de la misma ya que el aumento de peso por incorporación de agua a la masa del composite deteriora las propiedades mecánicas del mismo, lo cual con un tiempo de cepillado semejado in vitro de apenas tres meses no puede ser constatado, por lo que sería interesante evaluar este mismo estudio en un periodo de cepillado mayor y con la ayuda técnica de un método que nos permita determinar si hubo una expansión volumétrica por agua, es decir una expansión higroscópica ( Hux y col, 2003).

En cuanto a los especímenes que fueron sometidos a un proceso de pulido después de la polimerización del composite, este grupo mostró la mayor pérdida de masa, el pulido de las restauraciones dentales promueve la salud bucal al resistir la acumulación de restos de alimentos y bacterias patógenas, por la reducción de la rugosidad de la superficie del composite (Pontons y col, 2010) asumiendo que el proceso de pulido produjo grietas muy finas a nivel de la superficie de los especímenes por el desprendimiento de partículas de relleno, de tal forma que quedó expuesta la fase matriz de la resina la cual es la fase que es más susceptible al desgaste (Delgado, 2010), relacionando la pérdida generalizada de masa en este grupo, a que los especímenes fueron sometidos a dos procesos abrasivos, lo cuales fueron el proceso de pulido y el cepillado a través de la máquina deabrasividad.

El grupo de especímenes al cual se aplicó glicerina antes de la fotopolimerización del composite, se puede decir que es el método más aceptable desde un punto de vista e interpretación estadística, ya que siete de los once especímenes que perdieron peso, expresan una pérdida de peso de 2 miligramos como máximo, lo cual lo podríamos acoger como normal ya que dichos especímenes fueron sometidos a un proceso abrasivo severo. Uno de los doce especímenes aumentó de peso en alrededor 2 miligramos, lo cual se interpreta que menos del 9\% de los especímenes sufrieron sorción de agua (Mitra y col, 2003).

\section{Conclusiones}

En las condiciones que este estudio fue ejecutado puede concluirse que el empleo de matriz de celulosa, desencadeno el menor incremento de peso, el pulido post-fotopolimerización provocó una

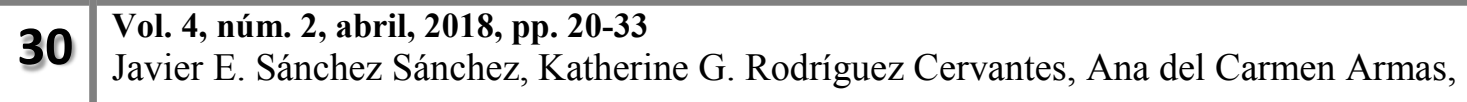
Iván R. García Merino, Henry S. Oñate-Negrete
} 
pérdida de peso de forma generalizada y crítica (con una media de 5,9 miligramos), el empleo de glicerina, desencadenó pérdida de peso, equivalente a $3,2 \mathrm{mg}$.

\section{Referencias Bibliográficas}

BRAGA R, FERRACANE J. 2004. Alternatives in polymerization contraction stress management. Crit. Rev. Oral. Biol. Med. 15 (3). Pp. 176-184.

CURY JA, TENUTA LMA, RIBEIRO CCC, PAES LEME AF. 2004. The importance of fluoride dentifrices to the current dental caries prevalence in Brazil. Braz Dent J. 15. Pp. 167-74.

HEINTZE SD, ZAPPINI G, ROUSSON V. 2005. Wear of ten dental restorative materials in five wear simulators--results of a round robin test. Dent Mater. 4. Pp. 304-317.

HEMINGWAY, C. A., PARKER, D. M., ADDY, M., BARBOUR, M. E. 2006. Erosion of enamel by non-carbonated soft drinks with and without toothbrushing abrasion. University dental hospital research laboratory in the UK. British Dental Journal. UK. 201 (7), Pp. 447-450.

HU X., MARQUIS P.M., SHORTALL A.C. 2003. Influence of filler loading on the two-body wear of a dental composite. J Oral Rehabil. 30 (7). Pp. 729-37.

IMFELD, T. ATTIN, T. 2008. Impacto of toothpaste slurry abrasivity and toothbrush filament stiffnes on abrasion of eroded enamel - an in vitro study. Acta odontologica Scandinava. 66. Pp. 231-235.

JUNG, M. 2005. Quiliti of bristle and end rouding on replaceable heads of powered toothbrushes. Jornal of Periodontology, Pp. 604-609.

KUIJS, R. FENNIS, W. KREULEN C. BARINK, M., VERDONSCHOT, N. 2003. Does Layering Minimize Shrinkage Stresses in Composite Restorations? J. Dent. Res.; 82 (12). Pp. 967-971.DE

\footnotetext{
31 Vol. 4, núm. 2, abril, 2018, pp. 20-33 Javier E. Sánchez Sánchez, Katherine G. Rodríguez Cervantes, Ana del Carmen Armas, Iván R. García Merino, Henry S. Oñate-Negrete
} 
SOUZA F. GUIMARAES R. SILVA C. A clinical evaluation of packable and microhybrid resin composite restorations: one-year report. Quintessence Int, 2005.

LITONJUA, L., ANDREANA, S., BUSH, P., COHEN, R. Desgaste Dentario:atrición, erosión, abrasión. Quintessence, 2005.

MACHADO, G., GONCALVES, E., RODRÍGUES, S., HENRIQUE, L. 2011. Effect of Whitening dentifrices on the surface roughness od comercial composites. Journal of Esthetic and Restorative Dentistry. Pp. 338-345.

MITRA S.B., WU D., HOLMES B.N. 2003. An application of nanotechnology in advanced dental materials. J Am Dent Assoc. 134 (10). Pp. 82-90.

PONTONS, J; ANTUNES, C.; MONDELLI, J. 2010. Evaluación del desgaste por cepillado, in vitro, de una resina compuesta, Fotopolimerizada por lámpara halógena, en dos tiempos diferentes. Acta odontológica. 48 (4).

RATTO, R.; DOS SANTOS RIBEIRO, D.; MARGARETE, M.; CUNHA, W.; CORRER, L. 2008. In vitro toothbrushing abrasion of dental resin composites: packable, microhybrid, nanohybrid and microfilled materials. Braz Oral Res. 22(2). Pp. 112-118.

RODRÍGUEZ G. DOUGLAS R., PEREIRA S. NATALIE A. 2008. Evolución y tendencias actuales en resinas compuestas. Acta odontológica. 46(3). Pp. 381-392.

SABBAGH, J. RYELANDT, L. BACHÉRIUS, L. BIEBUYCK, J. VREVEN, J. LAMBRECHTS, P. LELOUP, G. 2004. Characterization of the inorganic fraction of resin composite. Journal of Oral Rehabilitation. 31. Pp. 1090-1101.

SKRTIC, A. EICHMILLER, N. 2000. Physicomechanical evaluation of bioactive polymer composites based on hybrid amorphous calcium phosphates. Journal Biomedical Materials Research. (Applied Biomaterials). 53 (4). Pp. 381-391.

\footnotetext{
32 Vol. 4, núm. 2, abril, 2018, pp. 20-33 Javier E. Sánchez Sánchez, Katherine G. Rodríguez Cervantes, Ana del Carmen Armas, Iván R. García Merino, Henry S. Oñate-Negrete
} 
SÖDERHOLM KJ, LAMBRECHTS P, SARRETT D, ABE Y, YANG MC, LABELLA R, YILDIZ

E, WILLEMS G. 2001. Clinical wear performance of eight experimental dental composites over three years determined by two measuring methods. Eur J Oral Sci. 109. Pp. 273-281.

SOPARKAR, P. RUSTOGI, K., ZHANG, Y.P., PETRONE, M.E., DE VIZIO, W., PROSKING, H.M. 2004. Comparative tooth whitening and extrinsic tooth stain removal efficacy of two tooth whitening dentifrices: six - week clinical trial. Journal Clin. Dent. 15 (2). Pp. 46-51.

TEIXEIRA, E. 2005. In Vitro Toothbrush-Dentifrice Abrasiono, Two Restoirative Composites. Esthet Restor Dent. 17. Pp. 172-182.

TERRY DA, GELLER W. 2004. Selection defines design. J Esthet Restor Dent. 16. Pp. 213-225.

VERSTEEG, P.A., PISCAER, M., ROSEMA, N.A.M., TIMMERMAN, M.F., VAN DER VELDEN, U., VAN DER WEIJDEN, G.A. 2008. Evaluation of two soft manual toothbrushes with different filament designs in relation to gingival abrasion and plaque removing efficacy. Department of Periodontology Academic Centre for Dentistry Amsterdam. Int. Journal Dent. Hygiene. 6. Pp. 166173.

WANG L, GARCIA FC, AMARANTE DE ARAÚJO P, FRANCO EB, MONDELLI RF. 2004. Wear resistance of packable resin composites after simulated toothbrushing test. J Esthet Restor Dent; 16. Pp. 303-315.

WIEGAND, A., SCHWERZMANN, M., SENER, B., MAGALHÃES, A.C., ROOS, M., ZIEBOLZ, D., IMFELD, T., ATTIN, T. 2008. Impact of toothpaste slurry abrasivity and toothbrush filament stiffness on abrasion of eroded enamel - an in vitro study. Acta Odontol Scand. 66 (4). Pp. 231-235.

\footnotetext{
33 Vol. 4, núm. 2, abril, 2018, pp. 20-33 Javier E. Sánchez Sánchez, Katherine G. Rodríguez Cervantes, Ana del Carmen Armas, Iván R. García Merino, Henry S. Oñate-Negrete
} 